\title{
Novel technique of removal of broken intra-medullary nail from femur with secondary DCS plating
}

\author{
Neetin Pralhad Mahajan, Kartik Prashant Pande*, Pramod K. Bagimani
}

Department of Orthopaedics, Grant Government Medical College and Sir JJ group of Hospitals, Mumbai, Maharashtra, India

Received: 07 July 2021

Accepted: 13 August 2021

*Correspondence:

Dr. Kartik Prashant Pande,

E-mail: kartik.pande0393@gmail.com

Copyright: () the author(s), publisher and licensee Medip Academy. This is an open-access article distributed under the terms of the Creative Commons Attribution Non-Commercial License, which permits unrestricted non-commercial use, distribution, and reproduction in any medium, provided the original work is properly cited.

\begin{abstract}
Femoral shaft fractures are one of the commonest fractures of the lower limb which are frequently operated with intramedullary nailing which enables immediate post-operative mobilization of the patient. There could be various causes of nail breakage - some of the notable being weight bearing over the non-union of the femur shaft, or a re-trauma over the operated limb causing both the implant and the nail to be broken. There are various methods of removal of the broken implant the commonest being the use of T-reamer technique. However not always can this be used due to varied intra-operative obstacles in different cases as described in this case below. We have a 35 year old male patient who was brought to us 2 hours after an alleged history of slip and fall following which he had sustained right sided subtrochanteric femur fracture with a broken implant - intramedullary interlock nail. The patient is a previously operated case of right sided femur shaft fracture with interlocking nailing done 15 years back. The patient was operated with - broken implant removal on the right side along with a secondary DCS plating with bone grafting for the subtrochanteric femur fracture. Intra operative period was met with a certain number of challenges and difficulties in view of a 15 year old implant for removal which was successfully with removed with DCS plating done. As is obvious with the above case, it would be quite imperative to say that older the implant, more difficult it becomes for its removal.
\end{abstract}

Keywords: Implant removal, DCS plating, Subtrochanteric femur fracture, Bone grafting

\section{INTRODUCTION}

Femoral shaft fractures are one of the commonest fractures of the lower limb which are frequently operated with intramedullary nailing which enables immediate post operative mobilization of the patient. There could be various causes of nail breakage - some of the notable being weight bearing over the non-union of the femur shaft, or a re-trauma over the operated limb causing both the implant and the bone to be broken. There are various methods of removal of the broken implant the commonest being the use of T-reamer technique. ${ }^{1}$ The removal of a traditional hollow nail from the medullary cavity is deemed to be a bit easier than solid nails. There are various methods described for removal of a hollow nail which include use of hooks, guides wires or screws. ${ }^{2}$ However not always can this be used due to varied intra-operative obstacles in different cases as described in this case below.

\section{CASE REPORT}

We have a 35 years old male patient who was brought to us 2 hours after an alleged history of slip and fall following which he had sustained injury to the right hip. The patient is a previously operated case of bilateral femur interlock nailing done 15 years back. On preliminary imaging, on the right side - the patient was diagnosed to have a broken implant - the interlock nail along with a sub-trochanteric femur fracture at the same level (Figure 1). 
The patient was operated with - broken implant removal on the right side along with a secondary DCS plating for the sub-trochanteric femur fracture (Figure 2). Intra operative period was met with a certain number of challenges and difficulties i/v/o a 15 year old implant for removal which was successfully with removed with DCS plating with bone grafting done.

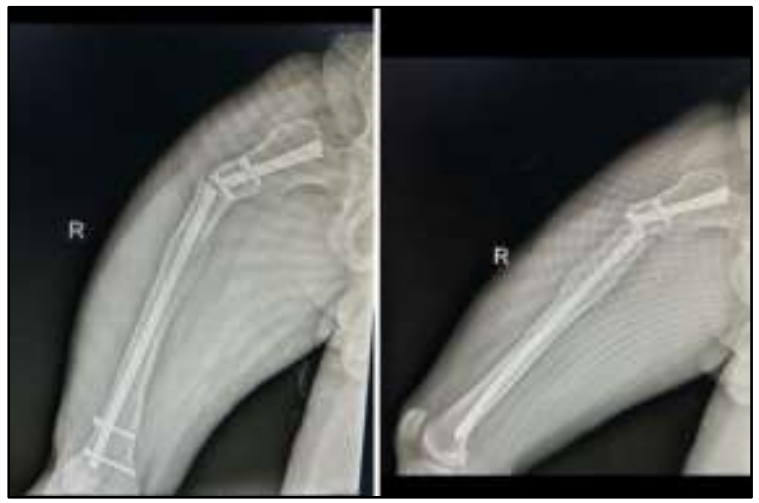

Figure 1: Pre-operative x-ray showing broken femur interlocking nail along with subtrochanteric femur fracture.

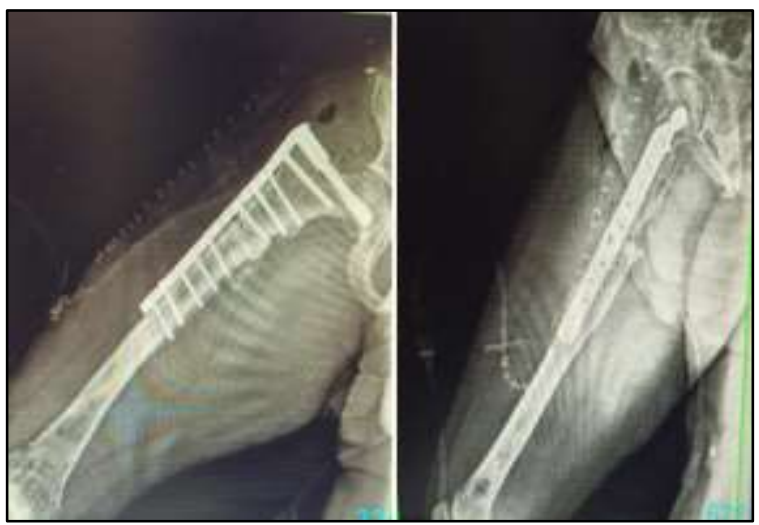

Figure 2: Immediate post-operative $x$-ray with implant removal done with secondary DCS plating with bone grafting.

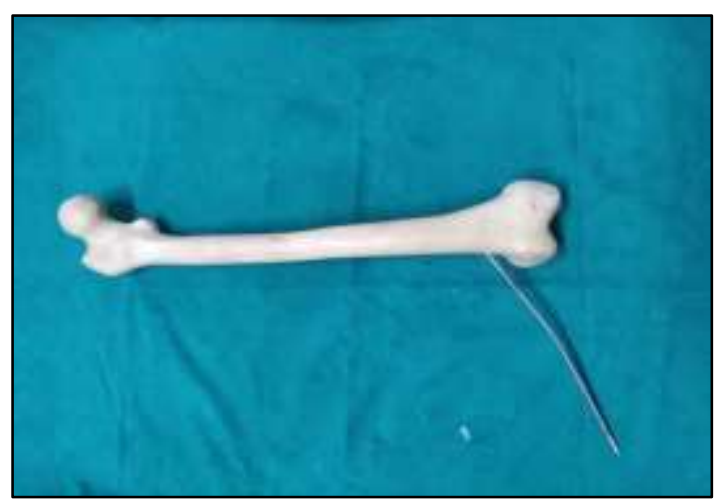

Figure 3: Cortical window made in the distal fragment in order to engage the bent Steinmann pin (AP view).

\section{Surgical technique}

The patient was placed in a supine position over the operating table. Induced under all aseptic precautions and under spinal and epidural anesthesia. Scrubbing, panting and draping was done. Incision was taken over the previous scar for the entry site.

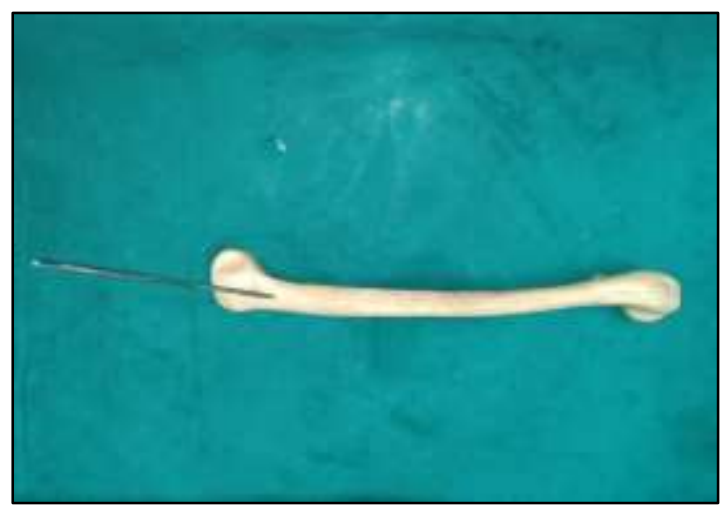

Figure 4: Cortical window in the distal femur to engage the Steinmann pin (lateral view).

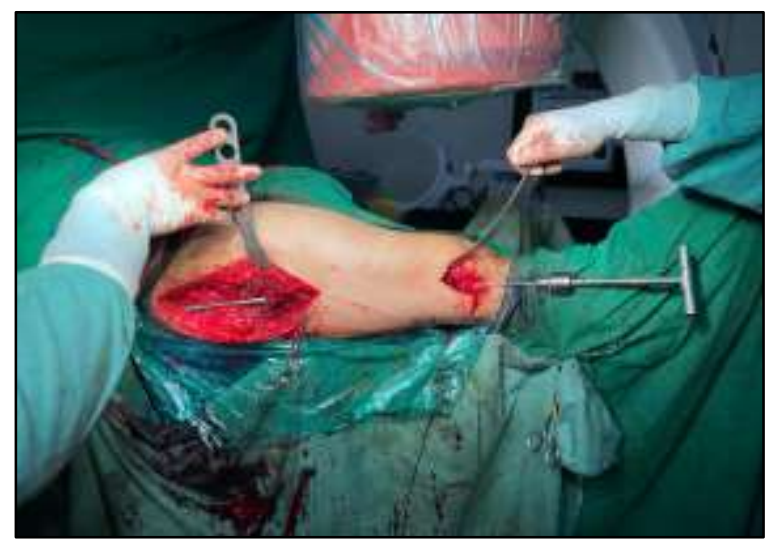

Figure 5: Distal fragment of the broken nail retrieved from the proximal fracture site after engaging the Steinmann pin distally.

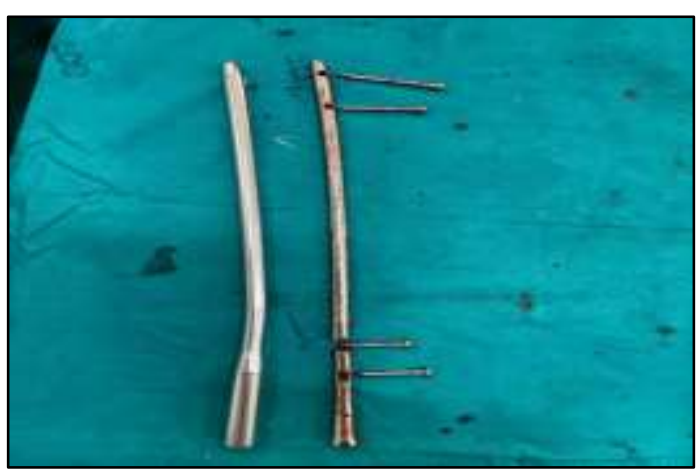

Figure 6: Removed broken femur interlock nail.

Due to extensive fibrosis over the entry point of femur, fracture site was opened over the previous proximal screw site incision and cortical screws were removed. The nail 
removal apparatus along with the nail-engager was first tried to get the broken proximal fragment of the nail retrieved. The proximal fragment was removed from the femur entry point from the tip of the greater trochanter after hammering it by engaging a long artery forceps from the fracture site into the nail. The major hurdle was encountered while removing the distal broken fragment of the nail. The distal fragment was removed from the fracture site. A significant amount of blood loss, as anticipated, was encountered while removal of both fragments of the implant. Initially, the removal was tried from the fracture site by engaging the nail removal reamer proximally.

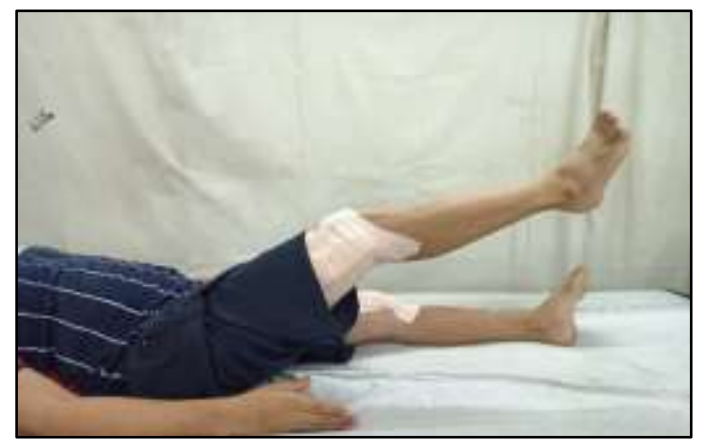

Figure 7: Straight leg raising on POD-2 showing hip flexion and knee extension.

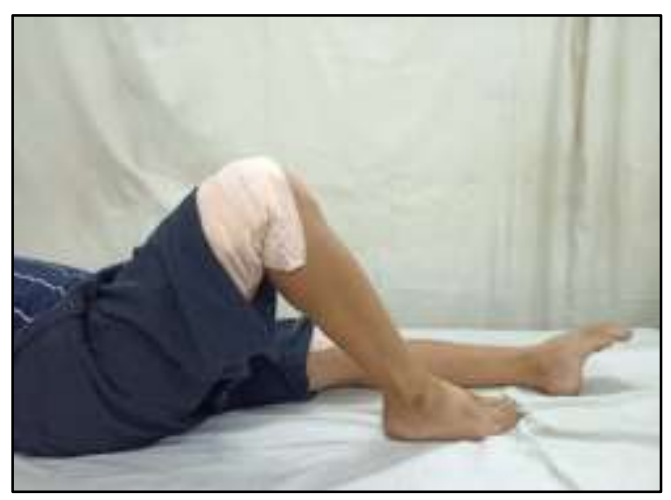

Figure 8: Hip and knee rom (flexion) on POD-2.

After a few attempts in vain, a unique technique was employed while removal of the distal fragment in the form of a Steinmann pin which was bent at the tip and engaged in the distal fragment after making a cortical window over the distal femur for retrieving the distal fragment (Figure $3,4)$. After engaging a Steinmann pin into the fragment, a T-handle was applied and by hammering technique, the distal fragment was retrieved from the fracture site proximally (Figure 5). After the implant removal was done (Figure 6), secondary procedure with fixation with a long cephalo-medullary nail was tried initially. It was encountered with difficulty as reaming was not possible due to extensive fibrosis and adhesions over the entry site. It was intra-op decided to fix the subtrochanteric femur fracture with Dynamic Condylar Screw with side plating with bone grafting was done with an acceptable reduction of the fracture. Post op period was unremarkable but the patient was shifted to CCU for vital monitoring $\mathrm{i} / \mathrm{v} / \mathrm{o}$ significant blood loss in the intra-op period. The patient was kept nil weight bearing over the right lower limb(operated with DCS) for 3 weeks. Hip and knee rom were started for the patient on POD2 (Figure 7,8) for early rehabilitation and to avoid post operative stiffness. The patient was later started and discharged on toe touch weight bearing for another 4 weeks.

\section{DISCUSSION}

Many a times, improper surgical technique, inexperience of the operating surgeon, low quality of implants, nonunion at the fracture site due to inadequate weight bearing or any other biological cause, recurrent trauma, and many such factors are responsible for the breakage of intramedullary nails. ${ }^{3}$ Removal of these broken nails often require longer operating time, greater blood loss, large amount of soft tissue and bone dissection, and use of special custom-made instruments. Broken intra-medullary nails often have to be removed by opening up the nonunion site [one of the common causes of nail removal], or in this case from the fracture site. The fracture site and the broken nail are at the same level in our case. Several methods have been described by various surgeons in removing the broken nail along with secondary fixation of the fracture. Many surgeons have advocated the use of the traditional T-reamer for removing the nail. ${ }^{4}$ Some of them have used custom made hooks to extract the distal fragment. Many different sizes of hooks are available for different sized nails. ${ }^{5}$ Metikala, described the removal of the nail using a ball-tipped guide wires. ${ }^{6}$ The major catch here depends upon the experience and surgical prowess of the operating surgeon in using the tools available in the operating room on table so as to remove the nail as quickly as possible so that the secondary implantation is completed as soon as possible so as to decrease the operative time. Here we have employed the use of a long artery forceps and a Steinmann pin by engaging it into the medullary cavity and extracting it from the respective ends (proximal fragment from the femur entry point and distal fragment from the fracture site situated proximally). This simple technique can be used for all cannulated as well as solid nails of varying internal nail diameters. It does not require any specialized instruments, is cost effective and can also be performed at centers with limited resources. Only limitation for the usage of such technique would be a very tight isthmus and nail interface. Due to many different types of nails and patterns of failure/non union/fractures, the surgeon should be aware of the different methods of nail extraction and the technique used in our case can be used as a useful addition to the existing techniques. ${ }^{7}$

\section{CONCLUSION}

As is obvious with the above case, it would be quite imperative to say that older the implant, more difficult it becomes for its removal. Most of the times, it depends on the skills and experience of the operating surgeon to 
employ different techniques and methods available along with various optimum intra-op instrument utilization for implant removal. The reason being the faster the implant removal, faster would be the secondary operative procedure, this case, DCS plating. And it is imperative employ various unique techniques intra-operatively so as to decrease the operative time so as to decrease the complications both intra and post operatively.

Funding: No funding sources

Conflict of interest: None declared

Ethical approval: Not required

\section{REFERENCES}

1. Pongsamakthai, Wanjak. Removal of the broken femoral nail with T-reamer technique: A three-case report. Journal of clinical orthopaedics and trauma. 2016;7(1):22-6.

2. Giannoudis PV, Matthews SJ, Smith RM. Removal of the retained fragment of broken solid nails by the intra-medullary route. Injury. 2001;32:407-10.
3. Vijay K, Singla A, Samarth M, Malhotra R. Removal of Broken Femoral Intramedullary Nail Using Arthroscopic Flipcutter. Techniques in Orthopaedics. 2014;29(3):171-3.

4. KL Pan. A Simple Method for Removal of the Distal Segment of a Broken Femoral Intramedullary Nail: A Case Report. Malaysian Orthopaedic Journal. 2012;6(3).

5. Franklin JL, Winquist RA, Benirschke SK, Hansen ST. Jr Broken intramedullary nails. J Bone Joint Surg Am. 1988;70:1463-71.

6. Metikala S, Mohammed R. Closed retrograde retrieval of the distal broken segment of femoral cannulated intramedullary nail using a ball-tipped guide wire. Indian J Orthop. 2011;45(4):347-50.

7. Lovell J, Krishna D, Gehr E. Stuffing the nail: A simple technique for the extraction of a broken femoral nail, Trauma Case Reports. 2017;9:38-41.

Cite this article as: Mahajan NP, Pande KP, Bagimani PK. Novel technique of removal of broken intra-medullary nail from femur with secondary DCS plating. Int J Res Orthop 2021;7:1039-42. 